PROCEEDINGS OF THE

AMERICAN MATHEMATICAL SOCIETY

Volume 126, Number 11, November 1998, Pages 3151-3159

S 0002-9939(98)04376-7

\title{
THE SKOLEM PROPERTY IN RINGS OF INTEGER-VALUED POLYNOMIALS
}

\author{
JEAN-LUC CHABERT, SCOTT T. CHAPMAN, AND WILLIAM W. SMITH \\ (Communicated by Wolmer V. Vasconcelos)
}

\begin{abstract}
Let $D$ be an integral domain with quotient field $K$ and $E \subseteq D$. We investigate the relationship between the Skolem and completely integrally closed properties in the ring of integer-valued polynomials$$
\operatorname{Int}(E, D)=\{f(X) \mid f(X) \in K[X] \text { and } f(a) \in D \text { for every } a \in E\} .
$$

Among other things, we show for the case $D=\mathbb{Z}$ and $|E|=\infty$ that the following are equivalent: (1) $\operatorname{Int}(E, \mathbb{Z})$ is strongly Skolem, (2) $\operatorname{Int}(E, \mathbb{Z})$ is completely integrally closed, and $(3) \operatorname{Int}(E, \mathbb{Z})=\operatorname{Int}(E \backslash\{a\}, \mathbb{Z})$ for every $a \in$ E.
\end{abstract}

\section{INTRODUCTION}

Throughout $D$ will be an integral domain with quotient field $K$. For a subset $E$ of $D$, we set

$$
\operatorname{Int}(E, D)=\{f(X) \mid f(X) \in K[X] \text { and } f(a) \in D \text { for every } a \in E\} .
$$

In the case $E=D$, instead of $\operatorname{Int}(D, D)$ we simply write $\operatorname{Int}(D)$. These rings of "integer-valued polynomials" were studied early by Pólya [17][18] and Ostrowski [16] and extensively more recently by various authors (see Brizolis [2], Cahen [4], Chabert [6, 7], McQuillan [13], and Gilmer [11]). These recent works center on certain algebraic properties of the rings and on conditions under which certain ideals are determined by their "values". The "Skolem conditions" [20] (as defined in the next section) and the complete integral closure are described in this work in terms of properties of the subset $E$. The results in some cases will reduce to already known results in the case $E=D([5]$ is a good general reference for such results).

\section{BASIC PROPERTIES}

In this section we establish some terminology and elementary observations regarding the properties to be discussed. Also, some simple examples are given which reflect the general results in the next section. If $I$ is an ideal of $\operatorname{Int}(E, D)$, then the ideal $I(a)=\{f(a) \mid f(X) \in I\}$ of $D$ where $a \in E$ is called the ideal of values of $I$

Received by the editors October 10, 1996 and, in revised form, March 25, 1997.

1991 Mathematics Subject Classification. Primary 13B25; Secondary 11S05, 12J10, 13E05, $13 \mathrm{G} 05$.

Key words and phrases. Integer-valued polynomial, Skolem property, Prüfer domain.

Part of this work was completed while the third author was on leave visiting Trinity University. 
at $a$. If $\Gamma$ is a set of ideals of $\operatorname{Int}(E, D)$ then consider the following conditions on elements $I$ and $J$ of $\Gamma$ with respect to $E$ :

(s) If $I(a)=D$ for all $a \in E$, then $I=\operatorname{Int}(E, D)$.

(S) If $I(a)=J(a)$ for all $a \in E$, then $I=J$.

Definition A. Let $R=\operatorname{Int}(E, D)$.

1. An ideal $I$ of $R$ is called unitary if $I \cap D \neq\{0\}$.

2. $R$ satisfies the $S k$ olem property with respect to $E$ if the set of finitely generated ideals of $R$ satisfies property ( $\mathrm{s}$ ).

3. $R$ satisfies the strong Skolem property with respect to $E$ if the set of finitely generated ideals of $R$ satisfies property (S).

It may be for two distinct subsets $E$ and $F$ of $D$ that $\operatorname{Int}(E, D)=\operatorname{Int}(F, D)$. If so, we will call $E$ and $F D$-polynomially equivalent. Hence, it may be necessary to denote the subset that is being considered for the Skolem property. It will follow from the results in Sections 3 and 4 in the case where $D$ is Dedekind with finite residue fields and $E$ and $F$ are d-subsets (see Definition $C$ below), that property (S) for finitely generated ideals of $R=\operatorname{Int}(E, D)$ depends on $R$ rather than on $E$. Indeed, in this case, if $R=\operatorname{Int}(E, D)$ satisfies $(\mathbf{S})$ with respect to $E$ and $\operatorname{Int}(F, D)=R$, then we will show in Corollary 4.7 that property (S) with respect to $F$ is satisfied since it is equivalent to $R$ being completely integrally closed. We do not know of an example of a more general domain $D$ and sets $E$ and $F$ where $\operatorname{Int}(E, D)=\operatorname{Int}(F, D)$ and the finitely generated ideals of $\operatorname{Int}(E, D)$ satisfy (s) or (S) with respect to $E$ but not with respect to $F$.

Definition B. 1. An element $a \in E$ is a polynomially isolated point of $E$ if $\operatorname{Int}(E, D)$ is properly contained in $\operatorname{Int}(E \backslash\{a\}, D)$.

2. A cofinite subset $F$ of $E$ (i.e., $E-F$ is finite) is a set with isolation if $\operatorname{Int}(E, D)$ is properly contained in $\operatorname{Int}(F, D)$.

Definition C. $E$ is a divisor subset of $D$ (or d-subset) if for polynomials $f(X)$, $g(X) \in K[X]$ with $f(a) \mid g(a)$ in $D$ for all except a finite number of $a \in E$, then $f(X) \mid g(X)$ in $K[X]$.

Throughout the remainder of the paper, by "isolated" we always mean "polynomially isolated". Definition C generalizes the notion of a d-ring given in [12]. Using our terminology, $D$ is a d-ring means that $D$ is a divisor subset of $D$.

If $D$ is Dedekind with finite residue fields, then the following three conditions are equivalent (see [11] and [15]):

(a) $\operatorname{Int}(E, D)=\operatorname{Int}(F, D)$,

(b) $E$ and $F$ represent the same system of residues of $D / P^{k}$ for each prime ideal power $P^{k}$,

(c) the topological closure of $E$ equals the topological closure of $F$ in the $P$-adic topology of $\hat{D}_{P}$ for all prime ideals $P$ of $D$.

With regard to Definition A, much attention has been given in the case $E=$ $D$ in Brizolis [2], Cahen [3, 4], Chabert $[6,7,8]$ and in a more general setting by McQuillan $[13,14,15]$. Under the general condition that $D$ is a Dedekind domain with finite residue fields, it has been established that $\operatorname{Int}(D)$ is Prüfer (and hence $\operatorname{Int}(E, D)$ is also Prüfer as it is an overring of $\operatorname{Int}(D)$ ). In this case, the Skolem and strong Skolem properties are established for $\operatorname{Int}(D)$ for certain $D$ $[2,3,4,6,7,8,13,14,15]$ and McQuillan shows that $\operatorname{Int}(E, D)$ satisfies the strong 
Skolem property for certain $E$. In this connection, it is also useful to note the works of Gilmer [11] and McQuillan [15] regarding the question of when $\operatorname{Int}(E, D)$ is the same as $\operatorname{Int}(F, D)$.

In the case where $D$ is a Dedekind domain with finite residue fields, the above cited works of Gilmer and McQuillan indicate that $a$ is an "isolated point of $E$ " if and only if for some prime ideal $P$ of $D, a$ is isolated in the topological sense as a point in $E$ in the topological space $\hat{D}_{P}$ (the P-adic completion of $D$ ). We note the definition given above is strictly algebraic and does not depend on this associated topological structure. With regard to this, it is easy to see in the presence of the topological structure that a cofinite set $F$ is a set with isolation if and only if it contains an isolated point. We do not know whether the same is true in the general algebraic setting. The following presents a strictly algebraic argument in the case $D$ is completely integrally closed (which of course would include the Dedekind case). We will use $\subset$ to denote proper set containment.

Theorem 2.1. Let $D$ be a completely integrally closed domain, $E$ a subset of $D$ and $F$ a cofinite subset of $E$. Then an element $a \in F$ is an isolated point of $E$ if and only if it is an isolated point of $F$.

Proof. If $a$ is an isolated point of $E$, then there is $f(X) \in \operatorname{Int}(E-\{a\}, D)$ such that $f(a) \notin D$. Obviously, $f(X) \in \operatorname{Int}(F-\{a\}, D)$ and $f(X) \notin \operatorname{Int}(F, D)$, that is, $a$ is an isolated point of $F$.

Conversely, assume that $a$ is not an isolated point of $E$. Let $E-F=\left\{b_{1}, \ldots, b_{n}\right\}$ and let $g(X)=\prod_{i=1}^{n}\left(X-b_{i}\right)$. Then, for each $f(X) \in \operatorname{Int}(F-\{a\}, D)$ and each $t \in \mathbb{N}, g(X) f(X)^{t} \in \operatorname{Int}(E-\{a\}, D)=\operatorname{Int}(E, D)$. Therefore, $g(a)(f(a))^{t} \in D$ for each $t \in \mathbb{N}$. Since $g(a) \neq 0, f(a)$ is almost integral over $D$, which is completely integrally closed. Therefore $f(a) \in D$ and $f(X) \in \operatorname{Int}(F, D)$.

Corollary 2.2. Let $D$ be a completely integrally closed domain and $E$ be a subset of $D$.

1. If $\left\{a_{1}, \ldots, a_{n}\right\}$ are all the isolated points of $E$, then $E-\left\{a_{1}, \ldots, a_{n}\right\}$ has no isolated points.

2. If $E-F=\left\{a_{1}, \ldots, a_{n}\right\}$, then $\operatorname{Int}(E, D) \subset \operatorname{Int}(F, D)$ if and only if some $a_{i}$ is an isolated point of $E$.

Proof. Assertion 1 is a direct consequence of the theorem. Let us prove assertion 2. If some $a_{i}$ is an isolated point of $E$, then $\operatorname{Int}(E, D) \subset \operatorname{Int}\left(E-\left\{a_{i}\right\}, D\right) \subseteq \operatorname{Int}(F, D)$. Conversely, if the $a_{i}$ are not isolated points of $E$, then it follows from the theorem that $\operatorname{Int}(E, D)=\operatorname{Int}\left(E-\left\{a_{1}\right\}, D\right)=\operatorname{Int}\left(E-\left\{a_{1}, a_{2}\right\}, D\right)=\cdots=\operatorname{Int}(F, D)$.

Example 2.3. Let $D=\mathbb{Z}$ and $E=\mathbb{P}$ be the set of prime integers. One can see "topologically" that each prime $p$ is an isolated point of $E$ in the $p$-adic integers $\hat{\mathbb{Z}}_{p}$. The above algebraic description of an isolated point is even easier to see as

$$
f(X)=\frac{(X-1) \cdots(X-p+1)}{p}
$$

is $\operatorname{in} \operatorname{Int}(\mathbb{P} \backslash\{p\}, \mathbb{Z})$ but not in $\operatorname{Int}(\mathbb{P}, \mathbb{Z})$. We also note that $f(X)$ is almost integral over $\operatorname{Int}(\mathbb{P}, \mathbb{Z})$ since for $g(X)=X-p$ one easily gets $g(X)(f(X))^{k} \in \operatorname{Int}(\mathbb{P}, \mathbb{Z})$ for all $k \geq 1$. Hence $\operatorname{Int}(\mathbb{P}, \mathbb{Z})$ is not completely integrally closed. The strong Skolem condition also fails since one can take $I=\langle g(X)\rangle$ and $J=\langle g(X), g(X) f(X)\rangle$. It is easy to see $I(q)=J(q)$ for every $q \in \mathbb{P}$, but $I \neq J$ since $g(X) f(X) \in I$ would imply 
$f(X) \in \operatorname{Int}(\mathbb{P}, \mathbb{Z})$. The ideas given in this example are described in the general situation in the next two sections, where it is shown for infinite subsets of $\mathbb{Z}$ that the conditions $\operatorname{Int}(E, \mathbb{Z})$ strong Skolem, $\operatorname{Int}(E, \mathbb{Z})$ completely integrally closed and $E$ has no isolated points, are equivalent. Additional information on $\operatorname{Int}(\mathbb{P}, \mathbb{Z})$ can be found in $[9,10]$.

The other definition given above, that of a d-subset, is also related to the completely integrally closed and Skolem properties of $\operatorname{Int}(E, D)$. McQuillan and Gunji [12] introduced the idea of a d-ring of which Definition $\mathrm{C}$ is a generalization. We remark that an obvious reformulation of the definition would state that $E$ is a d-subset whenever it has the property that the only rational functions $F(X)$ in $K(X)$ with $F(a) \in D$ for all except a finite number of $a$ in $E$ must be in $K[X]$. We also list the following lemma without proof since the first two statements are easy consequences of the definition and the third represents a result found in [11, Lemma 3] or [19, vol. II, p. 133].

Lemma 2.4. 1. If $E$ is a d-subset and $F$ is a cofinite subset of $E$, then $F$ is a $d$-subset.

2. If $E \subseteq F$ and $E$ is a d-subset, then $F$ is a $d$-subset. Hence, if $D$ contains a $d$-subset, then it must necessarily be a d-ring.

3. $E \subseteq \mathbb{Z}$ is a d-subset if and only if $|E|=\infty$.

In connection with 2 and 3 in the above lemma, it is of interest to consider the following example.

Example 2.5. Let $D$ be the ring of integers in a real quadratic number field. Set $E$ to be the set of units in $D$. We note in this case that we have $D$ is a Dedekind domain with finite residue fields and there is a fundamental unit $\lambda$ for which $E=\left\{ \pm \lambda^{n} \mid n \in \mathbb{Z}\right\}$. Hence $|E|=\infty$. Also, by Gunji and McQuillan [12, paragraph 1], $D$ is a d-ring. Although not of particular interest here, one can see that $E$ has no isolated points. This follows since for each prime ideal $P$ of $D$, each value in $E$ must be represented by an infinite number of distinct values of $E$ in $D / P^{t}$ (which is finite). However, $E$ is not a d-subset since the rational function $F(X)=\frac{1}{X}$ has the property that $F(a) \in D$ for every $a \in E$. It is also worth noting that $\operatorname{Int}(E, D)$ does not satisfy the Skolem property since $I=\langle X\rangle$ is an ideal of $\operatorname{Int}(E, D)$ for which $I(a)=D$ for all $a \in E$, yet $I \neq \operatorname{Int}(E, D)$. Theorem 3.1 of the next section will show that $\operatorname{Int}(E, D)$ is completely integrally closed.

In [13], McQuillan develops the strong Skolem property for $\operatorname{Int}(E, D)$ in the case $D$ is a Dedekind domain with finite residue fields which is a d-ring and where $E$ is a "homogenous" set. McQuillan's definition of a homogenous set $E$ is that there is a non-zero ideal $I$ of $D$ for which $a+I \subseteq E$ whenever $a \in E$. We relate this concept to our results in the following lemma.

Lemma 2.6. If $D$ is a Dedekind domain with finite residue fields which is a d-ring and $E$ is a homogenous subset of $D$, then

1. $E$ is a d-subset.

2. E has no isolated points.

Proof. $E$ homogenous means there is a nonzero ideal $I$ of $D$ so that $a+I \subseteq E$ for every $a \in E$. It is enough to assume there is a $m \neq 0$ in $D$ for which $a+m D \subseteq E$ in order to draw our conclusions. To see that $E$ is a d-subset, suppose $f(X) \in K(X)$ 
has the property that $f(b) \in D$ for all but finitely many $b \in E$. Letting $g(X)=$ $f(a+m X)$ we have $g(d) \in D$ for all but finitely many $d \in D$. Since $D$ is a d-ring, it follows $g(X) \in K[X]$ and hence $f(X) \in K[X]$. It remains to show that $E$ has no isolated points. This easily follows since for every prime ideal power $P^{k}$, there exists a nonzero $x \in I \cap P^{k}$. Thus, for $a \in E, a \neq a+x$ but $a \equiv a+x\left(\bmod P^{k}\right)$. As a result, $a$ cannot be an isolated point of $E$.

\section{The COMPlEtely INTEGRAlly ClOSED PROPERTY}

Recall that $\operatorname{Int}(D)$ is completely integrally closed if and only if $D$ is completely integrally closed (see [1, Proposition 7.2] or [5, Proposition VI.2.1]). For $\operatorname{Int}(E, D)$ things are a little more complicated. If $\operatorname{Int}(E, D)$ is completely integrally closed in its quotient field $K(X)$, then $D$ is completely integrally closed in $K$. With that hypothesis on $D$, we first establish necessary and sufficient conditions so that $\operatorname{Int}(E, D)$ is completely integrally closed.

Theorem 3.1. For a completely integrally closed domain $D$ the following are equivalent.

1. Int $(E, D)$ is completely integrally closed.

2. E has no isolated points.

Proof. (1) $\Rightarrow(2)$ Assume $a \in E$ and $f(X) \in \operatorname{Int}(E \backslash\{a\}, D)$. Then it easily follows $(X-a)(f(X))^{t} \in \operatorname{Int}(E, D)$ for $t \geq 1$. Since $\operatorname{Int}(E, D)$ is assumed to be completely integrally closed, we have $f(X) \in \operatorname{Int}(E, D)$. Therefore $\operatorname{Int}(E, D)=\operatorname{Int}(E \backslash\{a\}, D)$ and $E$ has no isolated points.

$(2) \Rightarrow(1)$ Let $f(X) \in K(X)$ be almost integral over $\operatorname{Int}(E, D)$. Since $\operatorname{Int}(E, D) \subseteq$ $K[X]$ which is completely integrally closed, we may assume $f(X) \in K[X]$. There exists $g(X) \neq 0$ in $\operatorname{Int}(E, D)$ so that $g(X)(f(X))^{t} \in \operatorname{Int}(E, D)$ for all $t \geq 1$. Let $a_{1}, \ldots, a_{n}$ be the (at most finite in number) zeros of $g(X)$. Then, for $b \in$ $E \backslash\left\{a_{1}, \ldots, a_{n}\right\}, g(b) \neq 0$ and $g(b)(f(b))^{t} \in D$ for $t \geq 1$. D is completely integrally closed, so this implies $f(b) \in D$. Hence $f(X) \in \operatorname{Int}\left(E \backslash\left\{a_{1}, \ldots, a_{n}\right\}, D\right)$ which is $\operatorname{Int}(E, D)$ by our assumption 2$)$ and Theorem 2.1. Therefore $\operatorname{Int}(E, D)$ is completely integrally closed.

The above proof can be modified to give an exact description of the complete integral closure of $\operatorname{Int}(E, D)$ which we now record as follows.

Theorem 3.2. For a completely integrally closed domain $D$ and subset $E$ of $D$, the complete integral closure $R^{*}$ of $R=\operatorname{Int}(E, D)$ is given by $R^{*}=\bigcup_{F \in \mathcal{F}} \operatorname{Int}(F, D)$ where $\mathcal{F}$ is the set of all cofinite subsets of $E$.

Proof. In the proof of Theorem 3.1, it was established that $f(X) \in R^{*}$ implies $f(X) \in \operatorname{Int}(F, D)$ for some $F \in \mathcal{F}$. Conversely, for $f(X) \in \operatorname{Int}(F, D)$ where $F=$ $E \backslash\left\{a_{1}, \ldots, a_{n}\right\}$ we have for $g(X)=\prod_{i=1}^{n}\left(X-a_{i}\right)$ that $g(X)(f(X))^{t} \in \operatorname{Int}(E, D)$ for all $t \geq 1$. Hence, $f(X) \in R^{*}$ which gives the indicated representation of $R^{*}$ in the theorem.

Corollary 3.3. If $D$ is completely integrally closed and $E$ is a subset of $D$ with only finitely many isolated points, then the complete integral closure of $R=\operatorname{Int}(E, D)$ is $R^{*}=\operatorname{Int}(F, D)$ where $F=E \backslash\{a \mid a$ is an isolated point of $E\}$. Moreover, in this case $R^{*}$ itself is completely integrally closed. 
Example 3.4. Consider as in Section 2 the domain $\operatorname{Int}(\mathbb{P}, \mathbb{Z})$ where $\mathbb{P}$ is the set of prime numbers. Taking $E=\mathbb{P} \cup\{1,-1\}$ we have [10, Proposition 2.1] that $\operatorname{Int}(\mathbb{P}, \mathbb{Z})=\operatorname{Int}(E, \mathbb{Z})$. Moreover, as indicated in Section 2, every $p \in \mathbb{P}$ is an isolated point of $E$ (notice that 1 and -1 are not). If $\mathbb{P}=\left\{p_{1}, p_{2}, \ldots\right\}$ is an enumeration of the primes in $\mathbb{P}$ and $F_{i}=E \backslash\left\{p_{1}, \ldots, p_{i}\right\}$, we have

$$
R=\operatorname{Int}(\mathbb{P}, \mathbb{Z}) \subseteq R^{*}=\bigcup_{i=1}^{\infty} \operatorname{Int}\left(F_{i}, \mathbb{Z}\right)
$$

We note the alternate description of $R^{*}$ is that

$$
R^{*}=\{f(X) \in \mathbb{Q}[X] \mid f(p) \in \mathbb{Z} \text { for all except a finite number of primes }\} .
$$

We also note that the containment $R^{*} \subset \operatorname{Int}(\{1,-1\}, \mathbb{Z})$ is proper. To see this, notice that $f(X)=\frac{X^{2}-1}{q}$ is in $\operatorname{Int}(\{1,-1\}, \mathbb{Z})$ for any integer $q$. For $q$ a prime, if $f(X)$ were to be in $R^{*}$, then $f(p) \in \mathbb{Z}$ for all but a finite number of primes. That is, $p^{2} \equiv 1(\bmod q)$ for all but a finite number of primes. If $q$ is chosen prime $>3$, then there are infinitely many primes $p$ of the form $p \equiv 2(\bmod q)$ (by Dirichlet's Theorem). Hence these primes cannot satisfy $p^{2} \equiv 1(\bmod q)$ which only holds for $p \equiv \pm 1(\bmod q)$.

In terms of the integer-valued polynomials we note that for a decreasing sequence of subsets $F_{1} \supseteq F_{2} \supseteq \cdots$ that

$$
\bigcup_{i=1}^{\infty} \operatorname{Int}\left(F_{i}, \mathbb{Z}\right) \subseteq \operatorname{Int}\left(\bigcap_{i=1}^{\infty} F_{i}, \mathbb{Z}\right)
$$

but the containment may be proper as is shown above. In fact, we argue $R^{*} \neq$ $\operatorname{Int}(Y, \mathbb{Z})$ for any $Y$, for if $R^{*} \subseteq \operatorname{Int}(Y, \mathbb{Z})$ we show $Y \subseteq\{1,-1\}$. Let $m \in \mathbb{Z}$ with $m \neq \pm 1$. Write $m=p_{1} \cdots p_{k}$ where the $p_{i}$ 's are not necessarily distinct primes. Let $c_{1}, \ldots, c_{\phi(m)}$ be a reduced system of residues modulo $m$ and set

$$
f(X)=\frac{\prod_{i=1}^{\phi(m)}\left(X-c_{i}\right)}{m} .
$$

Now, let $q$ be prime such that $q \notin\left\{p_{1}, \ldots, p_{k}\right\}$. Then $(q, m)=1$ and $q \equiv$ $c_{i}(\bmod m)$ for some $i$. Thus $f(q) \in \mathbb{Z}$ and $f(X) \in R^{*}$. But $f(m) \notin \mathbb{Z}$ and hence $Y \subseteq\{1,-1\}$. Our earlier observation that $R^{*} \subset \operatorname{Int}(\{1,-1\}, \mathbb{Z})$ completes the argument that $R^{*} \neq \operatorname{Int}(Y, R)$ for any $Y$.

\section{The Strong Skolem property}

We now turn our attention to the Skolem properties. The conditions that $E$ be a d-subset and have no cofinite sets with isolation are critical. Under sufficient hypothesis to insure that $\operatorname{Int}(E, D)$ is a Prüfer domain, we show these two conditions are sufficient to give the strong Skolem property (Theorem 4.6). We also show the conditions are necessary in the case where $D$ is Dedekind with finite residue fields (Theorem 4.3). We begin with a general property.

Lemma 4.1. If $\operatorname{Int}(E, D)$ is strongly Skolem, then $E$ has no subset with isolation; in particular $E$ has no isolated points.

Proof. Let $F=E \backslash\left\{a_{1}, \ldots, a_{r}\right\}$ be a cofinite subset of $E$, let $\varphi=\left(X-a_{1}\right) \cdots$ $\left(X-a_{r}\right)$, and let $f$ be in $\operatorname{Int}(F, D)$. In $\operatorname{Int}(E, D)$ consider the two ideals $I=\langle\varphi\rangle$ 
and

$$
J=\langle\varphi, \varphi f(X)\rangle
$$

noting, as we have before, that $\varphi f(X) \in \operatorname{Int}(E, D)$. It easily follows that $I(b)=J(b)$ for all $b \in E$. The strong Skolem property implies $I=J$, and hence $f(X) \in$ $\operatorname{Int}(E, D)$.

Lemma 4.2. Let $D$ be a Dedekind domain with finite residue fields and $E \subseteq D$. If $f(X), g_{1}(X), \ldots, g_{m}(X)$ are in $D[X], a$ is not an isolated point of $E, J$ is the ideal of $\operatorname{Int}(E, D)$ generated by $g_{1}(X), \ldots, g_{m}(X)$ and $f(b) \in J(b)$ for all except a finite number of $b$ in $E$, then $f(a) \in J(a)$.

Proof. The assumption that $a$ is not isolated and that $f(b) \in J(b)$ for almost all $b$ allows us for each prime ideal power $P^{k}$ to choose $b \in E$ with

1. $f(b) \in J(b)$ and

2. $b-a \in P^{k}$ (see (b) following Definition C).

For the case $J(a)=D$, the result is trivial, so we assume $J(a)=P_{1}^{n_{1}} \cdots P_{k}^{n_{k}}$. We complete the argument by establishing $f(a) \in P_{i}^{n_{i}}$ for all $i$ (hence $f(a) \in \bigcap P_{i}^{n_{i}}=$ $\left.\prod P_{i}^{n_{i}}=J(a)\right)$. For simplicity of notation, let $P^{n}=P^{n_{i}}$. Choose $b \in E$ with $f(b) \in J(b)$ and $b-a \in P^{n}$. Since $f(X), g(X)$ are in $D[X]$ we have the following: there exist $c_{1}, \ldots, c_{m}, d_{1}, \ldots, d_{m}, d$ in $D$ with

a) $f(b)=c_{1} g_{1}(b)+\cdots+c_{m} g_{m}(b)$,

b) $c_{j} g_{j}(b)-c_{j} g_{j}(a)=(b-a) d_{j}$ for every $j$, and Thus,

c) $f(b)-f(a)=(b-a) d$.

$$
f(a)=\sum_{j=1}^{m} c_{j} g_{j}(a)+(b-a)\left(\sum_{j=1}^{m} d_{j}-d\right) .
$$

Therefore, $f(a) \in J(a)+P^{n}=P^{n}$. Repeating the argument for $P^{n}$ being $P_{1}^{n_{1}}, \ldots$, $P_{k}^{n_{k}}$ yields $f(a) \in J(a)$.

Theorem 4.3. If $\operatorname{Int}(E, D)$ is strongly Skolem and $D$ is Dedekind with finite residue fields, then $E$ has no isolated points and is a d-set.

Proof. By Lemma 4.1, $E$ has no isolated points. To see that $E$ must be a $d$-set, assume $F(X) \in K(X)$ has the property that $F(a) \in D$ for almost all $a \in E$. Write $F(X)=\frac{f(X)}{g(X)}$ with $f(X), g(X) \in D[X]$ and consider the ideals $I=\langle g(X)\rangle$ and $J=$ $\langle f(X), g(X)\rangle$ of $\operatorname{Int}(E, D)$. Obviously $I(a) \subseteq J(a)$. Conversely, if $h(X) \in J$, then $h(a) \in I(a)$ for all $a \in E$ by Lemma 4.2 , since no $a$ is isolated in $E$. Hence $J(a)=I(a)$ for all $a \in E$. By the strong Skolem property for $\operatorname{Int}(E, D)$, it follows that $I=J$ and hence $f(X) \in\langle g(X)\rangle$ giving $F(X) \in K[X]$. Thus $E$ is a d-set.

The condition that $E$ is a d-subset enables us to conclude that when $\operatorname{Int}(E, D)$ is Prüfer it satisfies the Skolem property. The arguments given closely parallel those in McQuillan [13]. We repeat them because we do establish under the d-subset hypothesis that the Skolem condition is valid for a slightly larger class of ideals than the unitary ideals of McQuillan's discussion. Using this, we will establish under the additional Prüfer hypothesis the converse of Theorem 4.3. 
Theorem 4.4. If $E$ is a $d$-subset and $\operatorname{Int}(E, D)$ is Prüfer, then $\operatorname{Int}(E, D)$ satisfies the Skolem condition.

Proof. Assume $I=\left\langle f_{1}(X), \ldots, f_{n}(X)\right\rangle$ is a finitely generated ideal of $\operatorname{Int}(E, D)$ for which $I(a)=D$ for all $a \in E$. Since $\operatorname{Int}(E, D)$ is Prüfer, there exist $F_{1}(X), \ldots$, $F_{n}(X)$ in $I^{-1} \subseteq K(X)$ for which

$$
1=\sum_{i=1}^{n} f_{i}(X) F_{i}(X)
$$

Let $F$ be the cofinite subset of $E$ for which the $F_{i}(a)$ are defined for $a \in F$. Then, for a given $i$ and any $j$ with $1 \leq j \leq n, f_{j}(X) F_{i}(X) \in \operatorname{Int}(E, D)$, so $f_{j}(a) F_{i}(a) \in D$ for each $a \in F$. Therefore $I(a) \cdot F_{i}(a) \in D$ which implies $F_{i}(a) \in D$. Since $F_{i}(a) \in D$ for all except a finite number of $a \in E$ and $E$ is a d-subset, we have $F_{i}(X) \in K[X]$. Since $F_{i}(X) \in K[X], F=E$. Hence $F_{i}(X) \in \operatorname{Int}(E, D)$. This argument was for each $i=1,2, \ldots, n$ so we actually have $I^{-1} \subseteq \operatorname{Int}(E, D)$. Therefore $I=\operatorname{Int}(E, D)$.

Theorem 4.5. Let $E$ be a d-subset and $\operatorname{Int}(E, D)$ be Prüfer. If I and $J$ are finitely generated ideals with $I(a)=J(a) \neq\{0\}$ for all $a \in E$ then $I=J$.

Proof. By applying the condition first to $I \subseteq I+J$ and then to $J \subseteq I+J$, we see it is sufficient to establish the result in the case $I \subseteq J$. That is, we assume $I \subseteq J$ and $I(a)=J(a) \neq\{0\}$ for each $a \in E$. Write $I=J C$ and we have, since $I(\bar{a})=J(a) \neq\{0\}$, that $C(a)=D$ for all $a \in D$. By Theorem 4.4, $C=\operatorname{Int}(E, D)$ and hence $I=J$.

Notice that Theorem 4.5 generalizes [13, Lemma 2.6] since clearly unitary ideals satisfy the hypothesis of the theorem.

Theorem 4.6. Let $\operatorname{Int}(E, D)$ be Prüfer and $E$ be a d-subset which contains no sets with isolation. Then $\operatorname{Int}(E, D)$ is strongly Skolem.

Proof. As the argument indicates in the proof of Theorem 4.5, it is sufficient to establish the case $I \subseteq J$ where $I$ and $J$ are finitely generated ideals with $I(a)=J(a)$ for all $a \in E$. Choose $f(X) \in I$ to be a polynomial of least degree in $I$. Write $\langle f(X)\rangle=I A$ and $\langle f(X)\rangle=J B$ noting that both $A$ and $B$ are integral ideals of $\operatorname{Int}(E, D)$ with $B \subseteq A$. Now $I K[X]=f(X) K[X]$ so this gives $A K[X]=K[X]$, and hence $A \cap D \neq 0$. Let $F$ be the cofinite subset of $E$ which excludes the zeros of $f(X)$. For each $a \in F$, we have $I(a) A(a)=\langle f(a)\rangle=J(a) B(a)$ and hence $A(a)=B(a) \neq\{0\}$. It follows from Theorem 4.5 that $I \operatorname{Int}(F, D)=J \operatorname{Int}(F, D)$ since the cofinite subset $F$ of $E$ is also a d-subset of $D$, and hence that $I=J$ since $\operatorname{Int}(F, D)=\operatorname{Int}(E, D)$ by hypothesis.

We provide a useful summary of the above results. Recall the observation from Section 2 that in the classical case, $D=\mathbb{Z}$, any infinite subset of $\mathbb{Z}$ is a d-subset.

Corollary 4.7. Suppose $D$ is a Dedekind domain with finite residue fields and that $E \subseteq D$. The following statements are equivalent.

1. $E$ is a d-subset with no isolated points.

2. $\operatorname{Int}(E, D)$ is strongly Skolem.

3. $E$ is a d-subset and $\operatorname{Int}(E, D)$ is completely integrally closed. 
Corollary 4.8. Suppose $E \subset \mathbb{Z}$ and $|E|=\infty$. The following statements are equivalent.

1. E has no isolated points.

2. $\operatorname{Int}(E, \mathbb{Z})$ is strongly Skolem.

3. $\operatorname{Int}(E, \mathbb{Z})$ is completely integrally closed.

\section{REFERENCES}

[1] D.D. Anderson, D.F. Anderson and M. Zafrullah, Rings between $D[X]$ and $K[X]$, Houston Math. J. 17 (1991), 109-129. MR 92c:13014

[2] D. Brizolis, A theorem on ideals in Prüfer rings of integer-valued polynomials, Comm. Algebra 7 (1979), 1065-1077. MR 80j:13013

[3] P.-J. Cahen, Polynômes à valeurs entières, Thèse, Université Paris XI, Orsay, 1973.

[4] P.-J. Cahen, Integer-valued Polynomials on a Subset, Proc. Amer. Math. Soc. 117 (1993), 919-929. MR 93e:13011

[5] P.-J. Cahen and J.-L. Chabert, Integer-Valued Polynomials, Mathematical Surveys and Monographs, American Mathematical Society, Providence, 48 (1997). CMP 97:04

[6] J.-L. Chabert, Anneaux de "polynômes à valeurs entières" et anneaux de Fatou, Bull. Soc. Math. France 99 (1971), 273-283. MR 46:1780

[7] J.-L. Chabert, Un anneau de Prüfer, J. Algebra 107 (1987) 1-16. MR 88i:13022

[8] J.-L. Chabert, Une caractérisation des polynômes prenant des valeurs entières sur tous les nombres premiers, Canad. Math. Bull. 39 (1996), 402-407. MR 97h:11024

[9] J.-L. Chabert, S. Chapman and W. Smith, A Basis for the Ring of Polynomials IntegerValued on Prime Numbers to appear Factorization in Integral Domains, Marcel Dekker.

[10] J.-L. Chabert, S. Chapman and W. Smith, Algebraic Properties of the Ring of Polynomials Integer-Valued on Prime Numbers, Comm. Algebra. 25 (1997), 1945-1959. CMP 97:11

[11] R. Gilmer, Sets that determine integer-valued polynomials, J. Number Theory 33 (1989), 95-100. MR 90g:11142

[12] R. Gunji and D. McQuillan, On rings with a certain divisibility property, Michigan Math. J. 22 (1975), 289-299. MR 53:405

[13] D.L. McQuillan, On Prüfer domains of polynomials, J. Reine Angew. Math. 358 (1985), 162-178. MR 86k:13019

[14] D.L. McQuillan, Rings of integer-valued polynomials determined by finite sets, Proc. R. Ir. Acad. 85A (1985), 177-184. MR 87g:13016

[15] D.L. McQuillan, On a Theorem of R. Gilmer, J. Number Theory 39 (1991), 245-250. MR 92i: 13016

[16] A. Ostrowski, Über ganzwertige Polynome in algebraischen Zahlköpern, J. Reine Angew. Math. 149 (1919), 117-124.

[17] G. Pólya, Ueber ganzwertige ganze Funktionen, Rend. Circ. Matem. Palermo 40 (1915), 1-16.

[18] G. Pólya, Über ganzwertige Polynome in algebraischen Zahlköpern, J. Reine Angew. Math. 149 (1919), 97-116.

[19] G. Pólya and G. Szegö, Aufgaben und Lehrsätze aus der Analysis, Vol. II, Springer-Verlag, Berlin, 1925.

[20] T. Skolem, Ein Satz über ganzwertige Polynome, Norske Vid. Selsk (Trondheim) 9 (1936), 111-113.

Faculté de Mathématiques et d'Informatique, Université de Picardie, 33 rue Saint Leu, 80039 Amiens, France

E-mail address: jlchaber@worldnet.fr

Department of Mathematics, Trinity University, 715 Stadium Drive, San Antonio, Texas 78212-7200

E-mail address: schapman@trinity.edu

Department of Mathematics, The University of North Carolina at Chapel Hill, North Carolina 27599-3250

E-mail address: wwsmith@math.unc.edu 